\title{
Functional significance of the long non-coding RNA RP11-169D4.1 as a metastasis suppressor in laryngeal squamous cell carcinoma by regulating CDH1
}

\author{
JING ZHAO $^{1 *}$, KEXING LV $^{1 *}$, ZENG-HONG LI ${ }^{2}$, JIANHUI WU ${ }^{3}$, WEI GAO ${ }^{4}$, \\ THIAN-SZE WONG ${ }^{4}$, JIE LUO ${ }^{1}$, HAO QIN ${ }^{2}$, BIN WANG $^{5}$, QINGLING FU ${ }^{1}$ and WEN-BIN LEI ${ }^{1}$ \\ ${ }^{1}$ Department of Otolaryngology, The First Affiliated Hospital of Sun Yet-Sen University, Yuexiu, Guangzhou, \\ Guangdong; ${ }^{2}$ Department of Otolaryngology, The First People's Hospital of Foshan, Foshan, Guangdong; \\ ${ }^{3}$ The Otolaryngological Department, Meizhou People's Hospital, Meizhou, Guangdong; \\ ${ }^{4}$ Department of Surgery, The University of Hong Kong, Queen Mary Hospital, Hong Kong; \\ ${ }^{5}$ Department of Otolaryngology, The First People's Hospital of Guangzhou, Guangzhou, Guangdong, P.R. China
}

Received October 24, 2016; Accepted April 11, 2017

DOI: $10.3892 /$ or.2017.5645

\begin{abstract}
The present study investigated the expression profile and the function of RP11-169D4.1 and explored its potential mechanisms in laryngeal squamous cell carcinoma. The biological function of RP11-169D4.1 was examined using the MTT assay, flow cytometric analysis, wound healing and Transwell assays. The relationship between RP11-169D4.1 and miR-205-5p was discovered by Argonaute 2 protein immunoprecipitation. The target gene of RP11-169D4.1 was CDH1 which was assessed by Pearson's correlation analysis, RT-PCR and western blot assay. We demonstrated that RP11-169D4.1 expression was markedly decreased in LSCC tissues and cell lines. The overexpression of RP11-169D4.1 inhibited the proliferation, migration and invasion of LSCC cell lines as well as promoted apoptosis. We further verified that miR-205-5p had binding sites with RP11-169D4.1 and that RP11-169D4.1 could regulate the expression of $\mathrm{CDH} 1$. Ectopic transfection of RP11-169D4.1 led to a significant reduction in the downstream signaling molecule AKT in LSCC cells. The long non-coding RNA RP11-169D4.1 may serve as a tumor suppressor and a promising therapeutic target in laryngeal cancer, which could inhibit the process of EMT by regulating CDH1.
\end{abstract}

\section{Introduction}

Laryngeal carcinoma has been reported as the second most common head and neck squamous carcinoma, in which more

Correspondence to: Dr Wen-Bin Lei, Department of Otolaryngology, The First Affiliated Hospital of Sun Yet-Sen University, Yuexiu, Guangzhou, Guangdong, P.R. China

E-mail: leiwb@mail.sysu.edu.cn

${ }^{*}$ Contributed equally

Key words: RP11-169D4.1, miR-205-5p, CDH1, laryngeal squamous cell carcinoma, epithelial-mesenchymal transition than $95 \%$ of cases are laryngeal squamous cell carcinoma (LSCC) (1). This disease is a serious threat to patients' health and quality of life, especially for males, with a global incidence rate of 5.1/100,000 in 2008 (2,3). Advanced laryngeal carcinoma usually indicates poor treatment efficacy and a higher recurrence rate. Patients with invasion and metastasis generally have a much worse prognosis and have a 5-year survival rate of $\sim 60 \%$ (4). Therefore, more in-depth research of the molecular mechanisms may aid us in finding new diagnostic and/or therapeutic approaches to LSCC to improve the prognosis of LSCC patients (5).

Long non-coding RNAs (lncRNAs) usually range from $200 \mathrm{nt}$ to over $100 \mathrm{~kb}$ in length and are defined as endogenous cellular RNAs. At first, lncRNAs were discovered as 'transcriptional noise'. Currently, lncRNAs are considered to be a primary element of the human transcriptome. However, there is little knowledge regarding most of these lncRNAs, which require functional explanation (6). More new evidence has shown that in tumorigenesis and in cancer progression, lncRNAs play an important regulatory role (7). More lncRNAs have been discovered and identified as oncogenic or anti-oncogenic in head and neck cancer, including TUG1 (8), HOTAIR (9), ANRIL (10), CCAT2 (11), MEG3 (12), LOC285194 (13) and 91H (14). However, little is known about the role of long non-coding RNA in predicting metastasis and patient prognosis of LSCC. Furthermore, the underlying mechanisms of lncRNA in regulating LSCC metastasis remain unclear.

Microarray analysis of LSCC tissues showed abnormal expression of the lncRNA RP11-169D4.1. It was demonstrated that RP11-169D4.1 levels are significantly decreased in LSCC tissues, and decreased expression of RP11-169D4.1 indicates a poor prognosis and increased lymph node metastasis in patients with LSCC (15). However, the role of the lncRNA RP11-169D4.1 in LSCC remains unknown. In the present study, we further explored the role of the lncRNA RP11-169D4.1 and its potential underlying mechanism in LSCC. 


\section{Materials and methods}

Clinical specimens. A total of 51 patients with laryngeal squamous cell carcinoma were analyzed in the present study at the First Affiliated Hospital of Sun Yat-sen University (Guangzhou, China) between February 2012 and March 2014. All LSCC patients signed informed consent. The diagnosis of LSCC was histopathologically confirmed. Tumor and corresponding adjacent normal tissues were selected from each patient. Normal human laryngeal tissues were obtained at a minimum of $>10 \mathrm{~mm}$ from the edge of the cancerous area. Tissue samples were resected and immediately frozen in liquid nitrogen. They were stored at $-80^{\circ} \mathrm{C}$ until RNA extraction. The following clinicopathological data were collected: age, sex, tumor origin, TNM stage, lymph node metastasis, clinical stage and histological differentiation.

Cell culture. Human LSCC cell lines (SNU899 and SNU46) were obtained from Hong Kong University. All LSCC cells were maintained in Dulbecco's modified Eagle's medium (DMEM) with RPMI-1640, which was supplemented with $10 \%$ fetal bovine serum (FBS). All LSCC cells were incubated in a humidified incubator with $5 \% \mathrm{CO}_{2}$ at $37^{\circ} \mathrm{C}$.

RNA extraction. Total RNA was extracted using TRIzol ${ }^{\circledR}$ (Invitrogen, Carlsbad, CA, USA). A NanoDrop 1000 spectrophotometer (Thermo Fisher Scientific, Waltham, MA, USA) was used to confirm the RNA quality. The criterion of acceptable purity is an $\mathrm{OD}_{260 / 280}$ ratio of $\sim 1.8$. Reverse transcription was achieved with a First Strand cDNA Synthesis kit (Qiagen, Hilden, Germany) according to the manufacturer's protocol.

Transfection assays. The RP11-169D4.1 sequence (Gene: LINC01537, ENSG00000227467) was synthesized according to the full length RP11-169D4.1 sequence (based on the RP11-169D4.1 sequence) and then cloned into a pLVX vector (Invitrogen; Thermo Fisher Scientific). Then, either RP11169D4.1-pLVX or empty vector was transfected into LSCC cells using Lipofectamine 2000 reagent (Invitrogen; Thermo Fisher Scientific). The empty pLVX vector was used as the control.

Real-time quantitative RT-PCR. Real-time PCR was performed using the FastStart Universal Probe Master (Roche Applied Science, Indianapolis, IN, USA) on a LightCycler ${ }^{\circledR}$ 480 (Roche Applied Science). Primers for real-time PCR were purchased from Integrated DNA Technologies (Coralville, IA, USA). The detection probe was obtained from Roche Applied Science. The reaction was incubated at $95^{\circ} \mathrm{C}$ for $10 \mathrm{~min}$ followed by 55 cycles of $95^{\circ} \mathrm{C}$ for $15 \mathrm{sec}$ and $60^{\circ} \mathrm{C}$ for $1 \mathrm{~min}$. The mRNA was normalized to GAPDH levels using the $2^{-\Delta \Delta C t}$ method. The micro-RNA was normalized to U6 levels using the $2^{-\Delta \Delta C t}$ method.

Proliferation assay. LSCC cells $\left(1 \times 10^{4}\right)$ treated with either RP11-169D4.1-pLVX or empty vector were seeded into 96-well plates and cultured for 24, 48 and $72 \mathrm{~h}$. Before the indicated time-point, 3-(4,5-dimethylthiazol-2-yl)-2,5-diphenyltetrazolium bromide (MTT) $(0.5 \mathrm{mg} / \mathrm{ml}, \mathrm{pH} 4.7$; Sigma-Aldrich, St. Louis, MO, USA) was added for $4 \mathrm{~h}$. At the indicated times, the supernatant was removed and $150 \mu 1$ of dimethyl sulfoxide (DMSO) was added to the plate before shaking for $15 \mathrm{~min}$ at room temperature. A microplate reader (Thermo Fisher Scientific) was used to measure the absorbance at $490 \mathrm{~nm}$. The cell growth value was calculated from the mean values of 6 identical wells.

Flow cytometric analysis. LSCC cells $\left(2-5 \times 10^{5}\right)$ treated with either RP11-169D4.1-pLVX or negative control (NC) were seeded into 6-well plates. The cells were harvested by trypsinization after incubation for $48 \mathrm{~h}$. The cells were double stained with Annexin V and 7-AAD (Nanjing KeyGen Biotech, Co., Ltd., Nanjing, China) in the dark for $30 \mathrm{~min}$ at $37^{\circ} \mathrm{C}$. The cells were collected and analyzed on a flow cytometer (FACScan; BD Biosciences, Franklin Lakes, NJ, USA) to determine the apoptosis levels.

Wound healing assay. Transfected cells $\left(2-5 \times 10^{4} /\right.$ well) were cultured in 6-well plates and serum starved for $24 \mathrm{~h}$, after which the medium was replaced with medium containing serum (10\% FBS). A 100- $\mu$ l pipette tip was used to scratch the cell monolayer, which was imaged at 0 and $24 \mathrm{~h}$ after the wounding.

Cell invasion assays. Cells (2x10 $4 /$ well) in $200 \mu \mathrm{l}$ RPMI-1640 were seeded into the upper chamber of a Transwell apparatus with Matrigel (BD Biosciences) after transfection for $24 \mathrm{~h}$. The lower chambers were filled with media containing $15 \%$ FBS. The LSCC cells were incubated for $24 \mathrm{~h}$. After the cells invaded the membrane, they were fixed with $95 \%$ ethanol for $30 \mathrm{~min}$. Cells on the lower surface were stained with $0.1 \%$ crystal violet, photographed in three independent fields and counted.

Western blot analysis. Total protein was isolated from LSCC cells. A BCA protein quantification kit was used to determine the protein concentration. Proteins were separated on a $10 \%$ sodium dodecyl sulfate-polyacrylamide gel using SDS-PAGE and transferred electrophoretically onto polyvinylidene difluoride membranes (Whatman, Maidstone, UK). Then, the membranes were blocked for $1 \mathrm{~h}$ in $5 \%$ skim milk and washed three times with Tris-buffered saline containing $20 \%$ Tween-20 (TBST) at room temperature. The membranes were incubated with the primary antibodies overnight at $4^{\circ} \mathrm{C}$, washed the following day with TBST and incubated with secondary antibody for $1 \mathrm{~h}$ at room temperature. Finally, the immunoreactivity was visualized by enhanced chemiluminescence.

Mouse monoclonal anti-GAPDH (cat. no. KM9002; 1:5,000; Tianjin Sungene Biotech, Co., Ltd,. Tianjin, China), rabbit monoclonal anti-vimentin (cat. no. 5741S; 1:1,000; Cell Signaling Technology, Danvers, MA, USA), rabbit monoclonal E-cadherin (cat. no. A0965; 1:1,000; ABclonad, Inc., Seoul, Korea), rabbit monoclonal SNAIL2 (cat. no. A0572; 1:500; ABclonad), rabbit monoclonal AKT (cat. no. 4691S; 1:1,000; Cell Signaling Technology) and rabbit monoclonal p-AKT (cat. no. 4060S; 1:2,000; Cell Signaling Technology) primary antibodies were used. Goat anti-mouse IgG-HRP (cat. no. BA1050; 1:5,000; Wuhan Boster Bio-engineering, Co., Ltd., Wuhan, China) and goat anti-rabbit IgG-HRP (cat. no. 

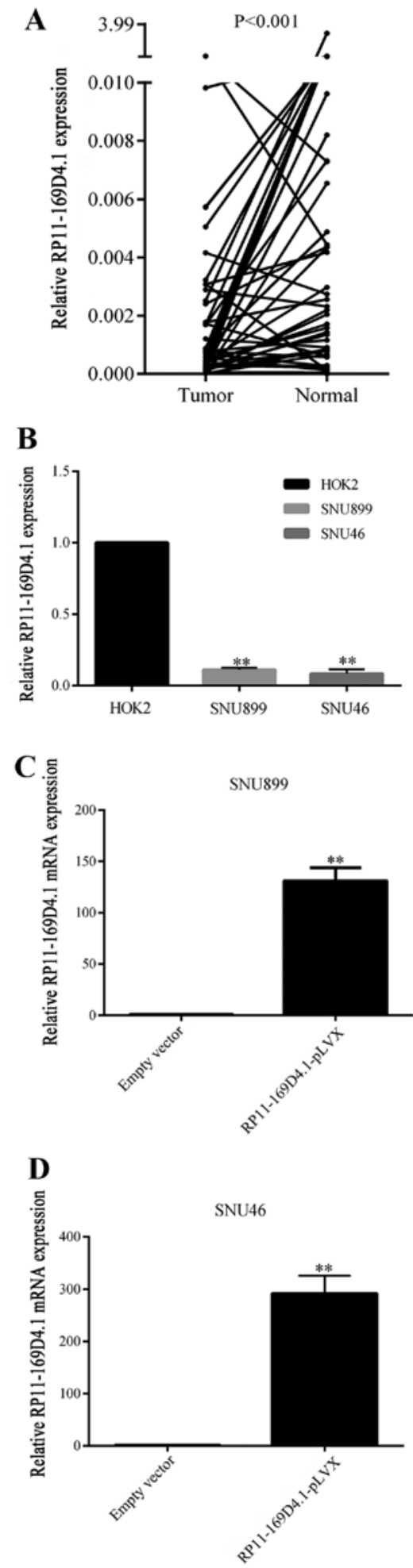

Figure 1. Analysis of RP11-169D4.1 expression in LCSS tissues and LSCC cell lines. (A) Relative expression of RP11-169D4.1 in LSCC tissues ( $\mathrm{n}=51)$ compared with adjacent normal tissues. RP11-169D4.1 expression was examined by RT-qPCR and normalized to GAPDH. (B) RP11-169D4.1 expression levels were determined by RT-qPCR in LSCC cell lines (SNU899 and SNU46) and the normal throat epithelial cell line (HOK). ${ }^{* *} \mathrm{P}<0.01$. (C and D) SNU899 and SNU46 cells were transfected with empty vector or RP11-169D4.1-pLVX for $48 \mathrm{~h}$, and the expression of RP11-169D4.1 was measured by qRT-PCR.

BA1055; 1:5,000; Wuhan Boster Bio-engineering) were used as secondary antibodies.
Table I. Relationship between RP11-169D4.1 expression and tumor clinicopathological features in LSCC.

\begin{tabular}{|c|c|c|c|c|}
\hline \multirow[b]{2}{*}{ Variables } & \multirow[b]{2}{*}{ Cases } & \multicolumn{2}{|c|}{$\begin{array}{c}\text { RP11-169D4.1 } \\
\text { expression }\end{array}$} & \multirow[b]{2}{*}{ P-value } \\
\hline & & $\begin{array}{l}\text { Low } \\
(26)\end{array}$ & $\begin{array}{l}\text { High } \\
(25)\end{array}$ & \\
\hline \multicolumn{5}{|l|}{ Sex } \\
\hline Male & 50 & 25 & 25 & 0.322 \\
\hline Female & 1 & 1 & 0 & \\
\hline \multicolumn{5}{|l|}{ Age (years) } \\
\hline$<60$ & 29 & 11 & 18 & $0.032^{\mathrm{a}}$ \\
\hline$\geq 60$ & 22 & 15 & 7 & \\
\hline \multicolumn{5}{|l|}{ T stage } \\
\hline $\mathrm{T} 1-2$ & 20 & 8 & 12 & 0.208 \\
\hline T3-4 & 31 & 18 & 13 & \\
\hline \multicolumn{5}{|l|}{ Lymph node } \\
\hline Positive & 20 & 14 & 6 & $0.029^{\mathrm{a}}$ \\
\hline Negative & 31 & 12 & 19 & \\
\hline \multicolumn{5}{|l|}{ Clinical stage } \\
\hline Early & 16 & 6 & 10 & 0.193 \\
\hline Advance & 35 & 20 & 15 & \\
\hline \multicolumn{5}{|l|}{ Histological differentiation } \\
\hline $\begin{array}{l}\text { Well and moderately } \\
\text { differentiated }\end{array}$ & 29 & 13 & 16 & 0.313 \\
\hline Poor and undifferentiated & 22 & 13 & 9 & \\
\hline \multicolumn{5}{|l|}{ CDH1 expression } \\
\hline Low & 26 & 17 & 9 & $0.036^{\mathrm{a}}$ \\
\hline High & 25 & 9 & 16 & \\
\hline \multicolumn{5}{|c|}{ The correlation between RP11-169D4.1 and CDH1 } \\
\hline Pearson correlation & 0.744 & & & \\
\hline P-value & $<0.001^{\mathrm{b}}$ & & & \\
\hline
\end{tabular}

Argonaute 2 (AGO2) protein immunoprecipitation. LSCC cells were transfected with either miR-205-5p inhibitor or negative control (Qiagen) using the Lipofectamine 2000 reagent. Subsequently, a human Argonaute 2 (Ago2) miRNA isolation kit (Wako Pure Chemical Industries, Osaka, Japan) was used to isolate the Ago2 complex. After transfection, the cells were lysed, and anti-Ago2 monoclonal antibody-immobilized beads (Wako Pure Chemical Industries) were added to the cell lysate. After incubation for $2 \mathrm{~h}$ at $4^{\circ} \mathrm{C}$, the beads were washed with wash buffer, and the Ago 2 complex was eluted from the beads. The levels of miR-205-5p and RP11-169D4.1 were measured from the eluted Ago 2 complex. The PCR primer-probe pairs for RP11-169D4.1 quantification were as follows: forward primer, 5'-CCGGAATTCCCCAGACACAG GGCAGCCTTCC-3' and reverse primer, 5'-ATAAGAATG CGGCCGCTTTTATATAATATTTTGAAT-3'; probe, \#15 from the Universal Probe Library (Roche Applied Science). The PCR primer-probe pair for miR-205-5p quantification was 
A

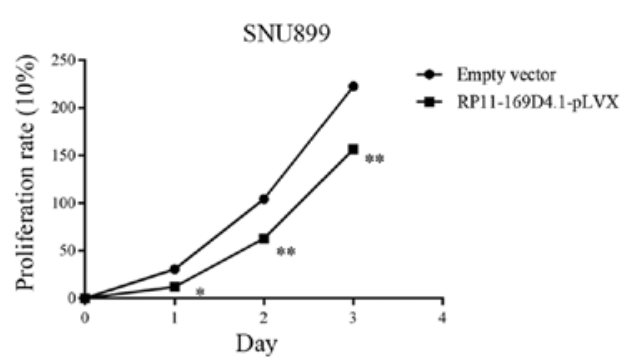

C
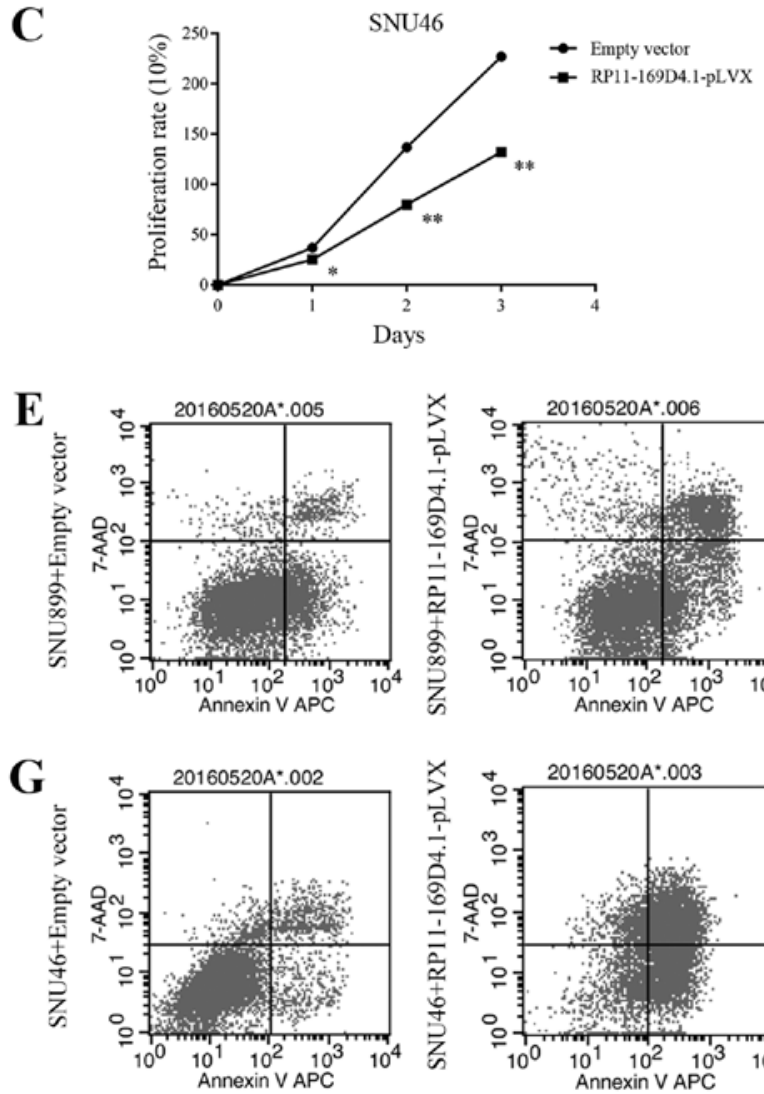
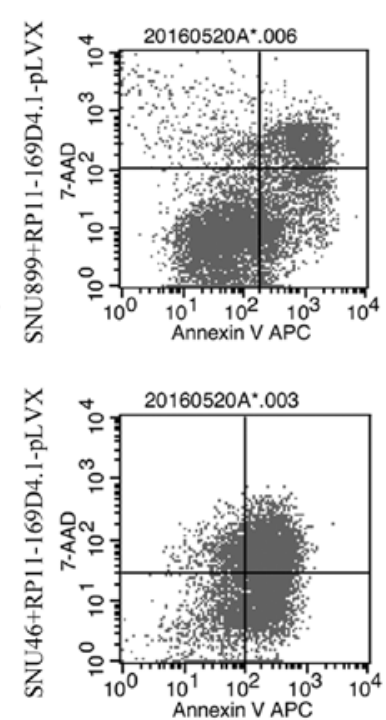

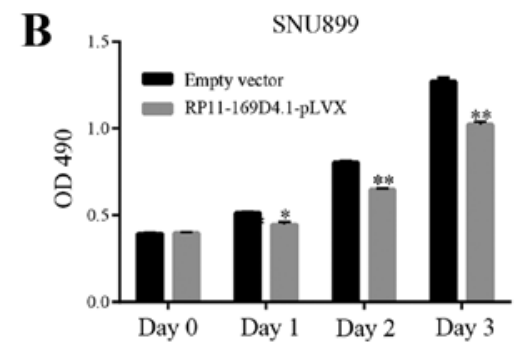

D

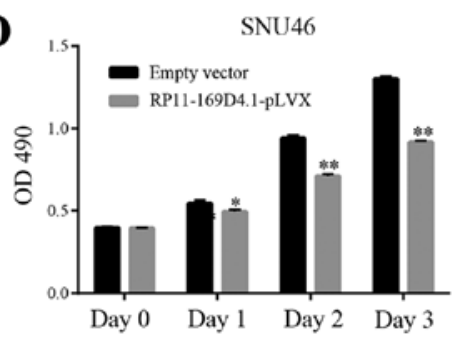

$\mathbf{F}$

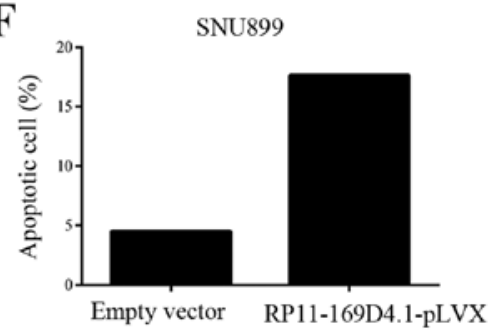

$\mathbf{H}$

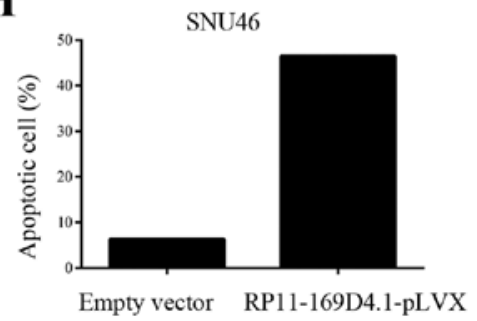

Figure 2. RP11-169D4.1 suppresses LSCC cell proliferation and promotes apoptosis. Overexpression of RP11-169D4.1 decreased the (A-D) SNU899 and SNU46 cells growth. ${ }^{*} \mathrm{P}<0.05,{ }^{* *} \mathrm{P}<0.01$. At $48 \mathrm{~h}$ after transfection, cell apoptosis was analyzed by flow cytometry. Late apoptotic cells increased significantly after $48 \mathrm{~h}$ transfection with RP11-169D4.1-pLVX compared to controls in (E and F) SNU899 and (G and H) SNU46 cells.

as follows: 5'-TCCTTCATTCCACCGGAGTCTG-3'; probe \#61. The expression level of glyceraldehyde-3-phosphate dehydrogenase $(G A P D H)$ was quantified using the Universal Probe Library Human GAPD Gene Assay (Roche Applied Science).

Statistical analysis. Data were reported as the means \pm standard deviation from at least three independent experiments. All of the statistical analyses were performed using the SPSS 20.0 statistical software (IBM, New York, NY, USA) with either Student's t-test (two tailed) or one-way analysis of variance (ANOVA) for multiple groups. Differences were considered statically significant at the probability of $\mathrm{P}<0.05$.

\section{Results}

RP11-169D4.1 expression is downregulated in LSCC tissues and cell lines. The expression levels of lncRNA RP11169D4.1 were evaluated by qRT-PCR in 51 paired LSCC tissues and adjacent normal tissues. As shown in Fig. 1A,
RP11-169D4.1 expression was much lower in LSCC tissues than that in normal tissues $(\mathrm{P}<0.001)$. The clinicopathological characteristics of the 51 patients, including age, sex, tumor origin, TNM stage, lymph node metastasis, clinical stage and histological differentiation are presented in Table I. LncRNA RP11-169D4.1 expression in cancer tissues was associated with lymph node metastasis $(\mathrm{P}=0.029)$. The expression of RP11-169D4.1 was significantly downregulated in LSCC cell lines compared with normal throat epithelial cells as shown in Fig. 1B.

RP11-169D4.1 suppresses proliferation and promotes apoptosis in LSCC cells. To investigate the role of RP11-169D4.1 in the regulation of cell proliferation and apoptosis, SNU899 and SNU46 cells were transfected with RP11-169D4.1-pLVX. qRT-PCR was used to measure the expression of RP11-169D4.1, which was greatly increased (Fig. 1C and D).

The growth curves determined by the MTT assay showed that overexpression of RP11-169D4.1 could suppress the 
A
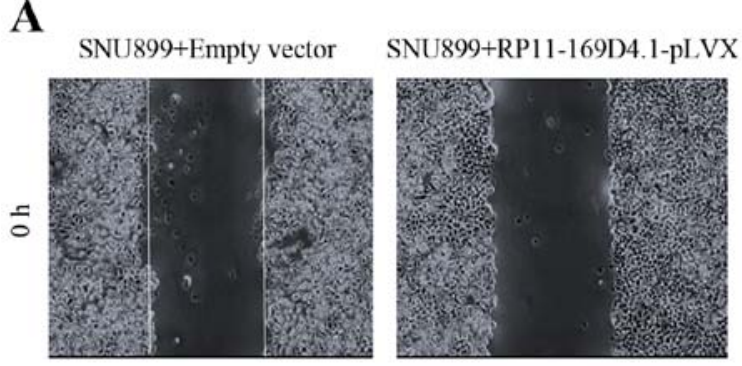

B

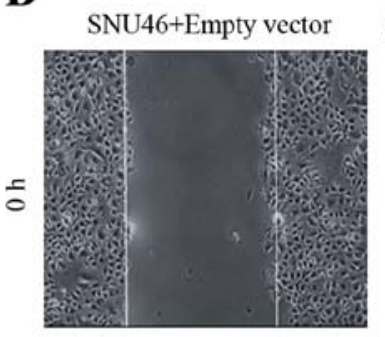

SNU46+RP11-169D4.1-pLVX

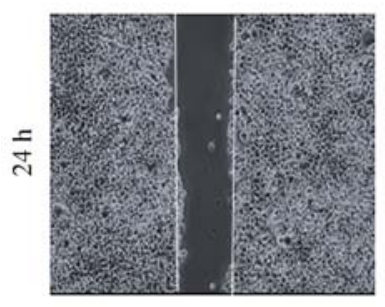

C

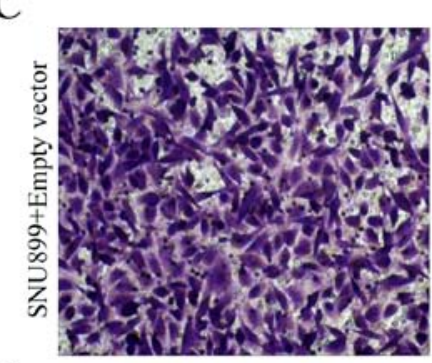

E

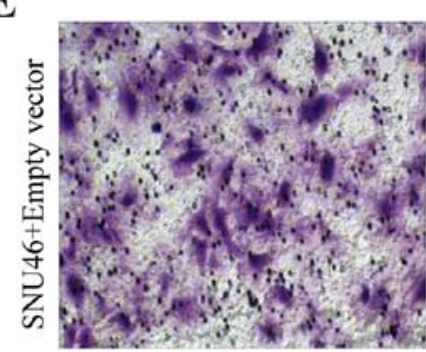

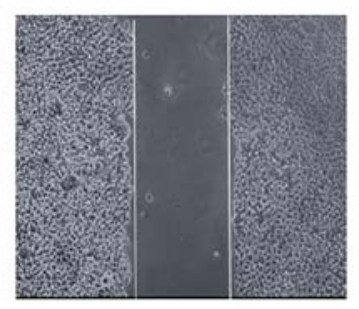
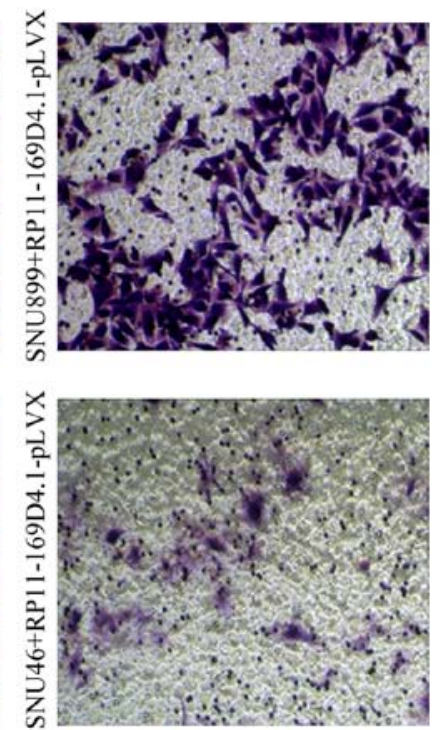

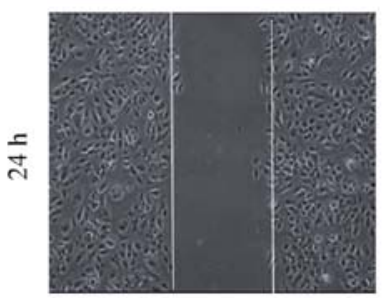

D

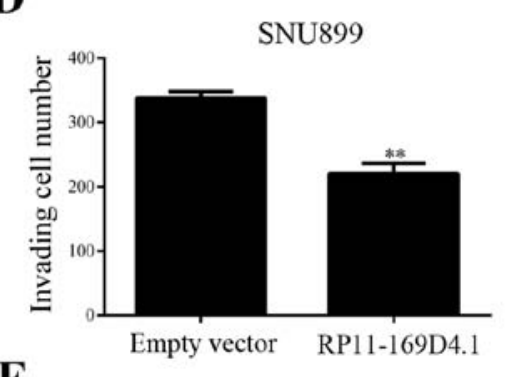

F

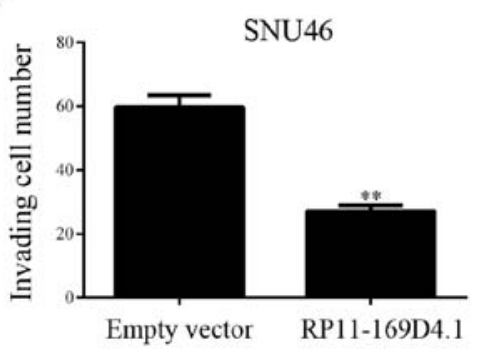

Figure 3. RP11-169D4.1 suppresses LSCC cell migration and invasion. Wound healing assay shows that SNU899 (A) and SNU46 (B) cell motility could be effectively suppressed by RP11-169D4.1-pLVX compared by empty vector. Upregulation of RP11-169D4.1 in SNU899 (C and D) and SNU46 (E and F) significantly inhibited invasion compared with the control group. ${ }^{*} \mathrm{P}<0.05,{ }^{* *} \mathrm{P}<0.01$.

proliferation of SNU899 and SNU46 cells at 24, 48 and $72 \mathrm{~h}$ after transfection (Fig. 2A-D). The apoptosis assay showed that the percentage of apoptotic cells was significantly increased in response to RP11-169D4.1 overexpression compared with NC overexpression in SNU899 and SNU46 cells (Fig. 2E-H). These results indicated the anti-proliferative and pro-apoptotic role of RP11-169D4.1 in LSCC cells.

RP11-169D4.1 inhibits migration and invasion in LSCC cells. To examine the effect of RP11-169D4.1 on migration and invasion of LSCC cells, wound healing and Transwell invasion assays were conducted. We found that LSCC cells transfected with RP11-169D4.1-pLVX showed less wound closure than cells transfected with empty pLVX vector (Fig. 3A and B). The Transwell assays showed that overexpression of RP11-169D4.1 inhibited the invasion of LSCC cells transfected with RP11-169D4.1-pLVX (Fig. 3C-F). These results suggest that RP11-169D4.1 contributes to the inhibition of the migratory and invasive capacity of LSCC cells.
RP11-169D4.1 inhibits EMT in LSCC cells. To determine whether overexpression of RP11-169D4.1 inhibits epithelialmesenchymal transitions (EMT) in LSCC cells, we enhanced RP11-169D4.1 expression in SNU899 and SNU46 cells and examined the mRNA expression of EMT markers by RT-PCR and the protein levels by western blot assay. As illustrated in Fig. 4, the level of CDH1 was improved, and the levels of Snail2 and vimentin were reduced in cells transfected with RP11169D4.1-pLVX. These results showed that RP11-169D4.1 was able to inhibit EMT in LSCC cells.

RP11-169D4.1 was targeted and inhibited by miR-205-5p. Given the observation that RP11-169D4.1 played an important role in regulating the biological properties of LSCC cells, we next investigated the potential mechanisms of RP11-169D4.1 using the bioinformatics tool RNA22 (16). RNA22 predicted that RP11-169D4.1 was a target of miR-205-5p (Fig. 5A). We transfected LSCC cells with either a miR-205-5p inhibitor or miR-NC and confirmed the transfection efficiency using 


\section{A}
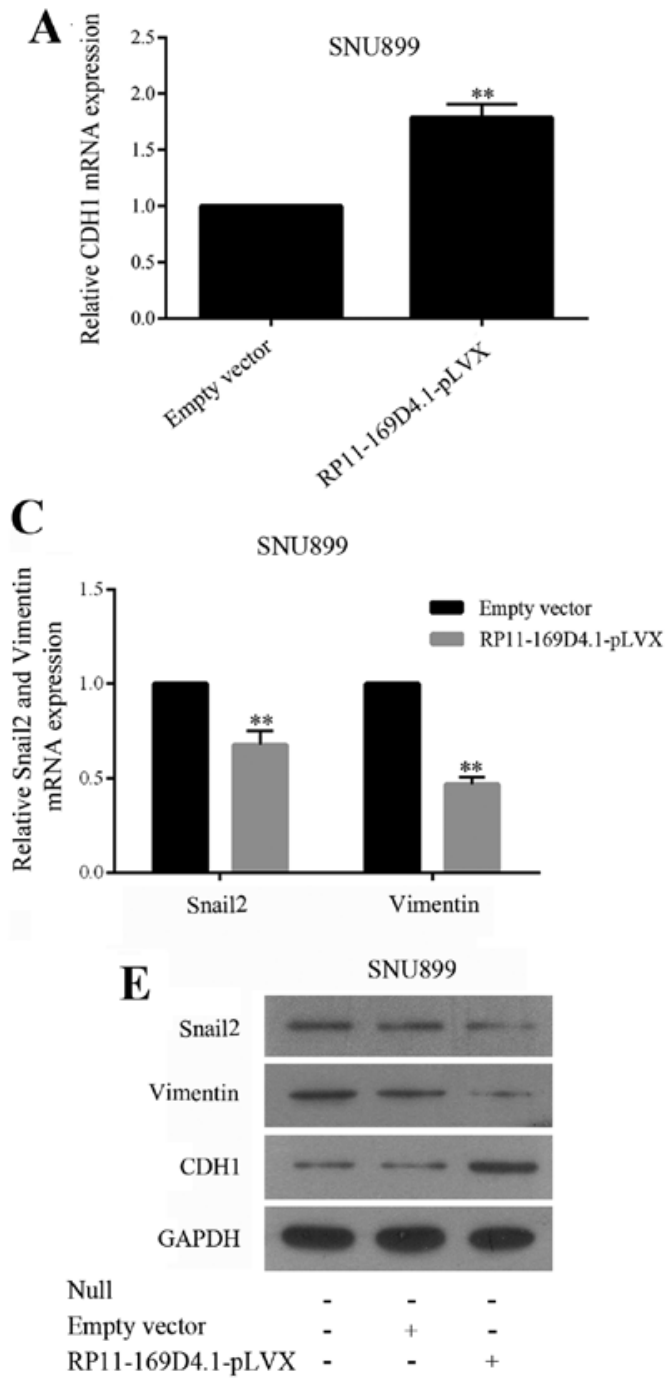

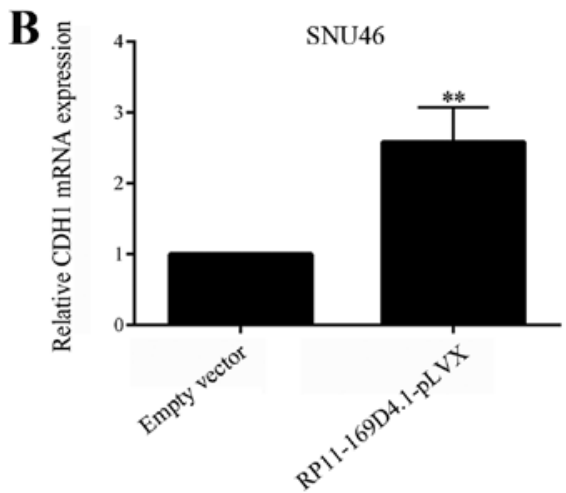

D
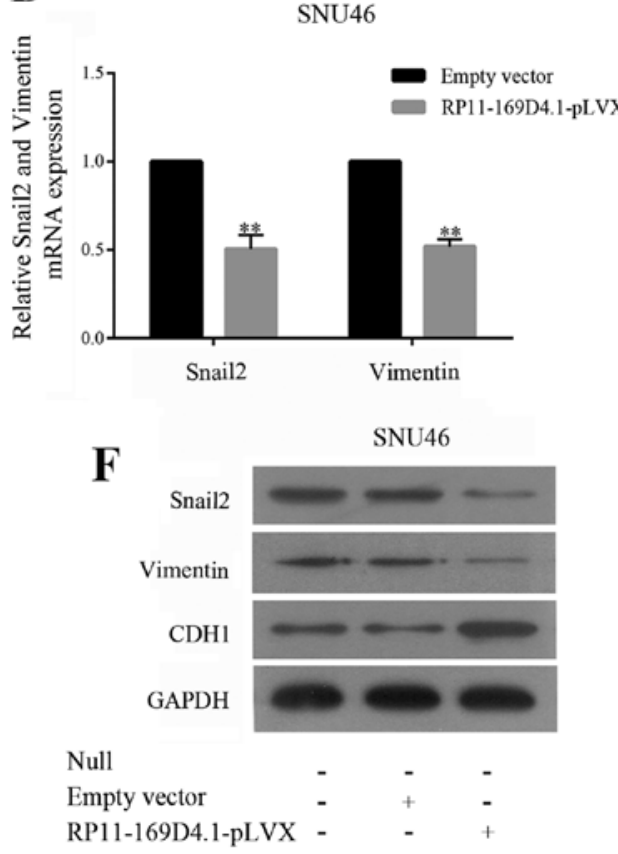

Figure 4. qRT-PCR analysis of CDH1 (A and B), Snail2 and vimentin (C and D) mRNA levels in SNU899 and SNU46 cells following RP11-169D4.1 overexpression. (E and F) Western blot analysis of CDH1, Snail2 and vimentin protein levels in SNU899 and SNU46 cells following RP11-169D4.1 overexpression. ${ }^{*} \mathrm{P}<0.05,{ }^{* *} \mathrm{P}<0.01$.

RT-PCR. SNU899 and SNU46 cells with low expression of miR-205-5p showed higher levels of RP11-169D4.1 compared with the negative control cells (Fig. 5D). However, the overexpression of RP11-169D4.1 did not affect the expression of miR-205-5p in LSCC cells (Fig. 5E), which indicates that miR-205-5p might inhibit the expression of RP11-169D4.1.

We performed immunoprecipitation of endogenous protein-mRNA complexes in SNU899 and SNU46 cells. Both miR-205-5p and RP11-169D4.1 were enriched in the immunopurified AGO2 complex, suggesting that RP11-169D4.1 is an AGO2-selected transcript in the LSCC cell lines. In the cell lines transfected with the miR-205-5p inhibitor, the transcript level of mature miR-205-5p and RP11-169D4.1 dropped significantly compared with the cell lines transfected with miR-NC (Fig. 5B and C). The results indicated that mature miR-205-5p could possibly bind to the RP11-169D4.1 transcript and hinder RP11-169D4.1 expression by 3'-UTR-mediated mRNA degradation.

CDH1 expression is downregulated and correlated with RP11169D4.1 in LSCC tissues. CDH1, also known as E-cadherin, is a well-established tumor suppressor (17-19). Loss of CDH1 can trigger EMT and also has a strong association with the invasive metastasis of various tumors $(20,21)$. By using the Oncomine StarBase, large sets of data that show reduced CDH1 mRNA levels in various cancerous tissues compared to normal tissues can be searched (Fig. 6). To determine the correlation between RP11-169D4.1 and CDH1, we further analyzed the expression of CDH1 in 51 paired LSCC tissues and adjacent normal tissues. CDH1 was identified as having lower expression in LSCC tissues than in normal tissues $(\mathrm{P}=0.038$; Fig. 7A). In addition, the correlation analysis revealed that there was a positive correlation between the expression of RP11-169D4.1 and CDH1 $\left(\mathrm{P}<0.001 ; \mathrm{R}^{2}=0.744\right)$ (Table I; Fig. 7B).

As shown in Fig. 4, the upregulation of RP11-169D4.1 could enhance the level of CDH1 protein. Furthermore, we found that the expression of CDH1 mRNA was significantly improved in the cells transfected with RP11-169D4.1-pLVX compared to cells transfected with empty vector. These results indicated that RP11-169D4.1 could modulate the expression of $\mathrm{CDH} 1$. 

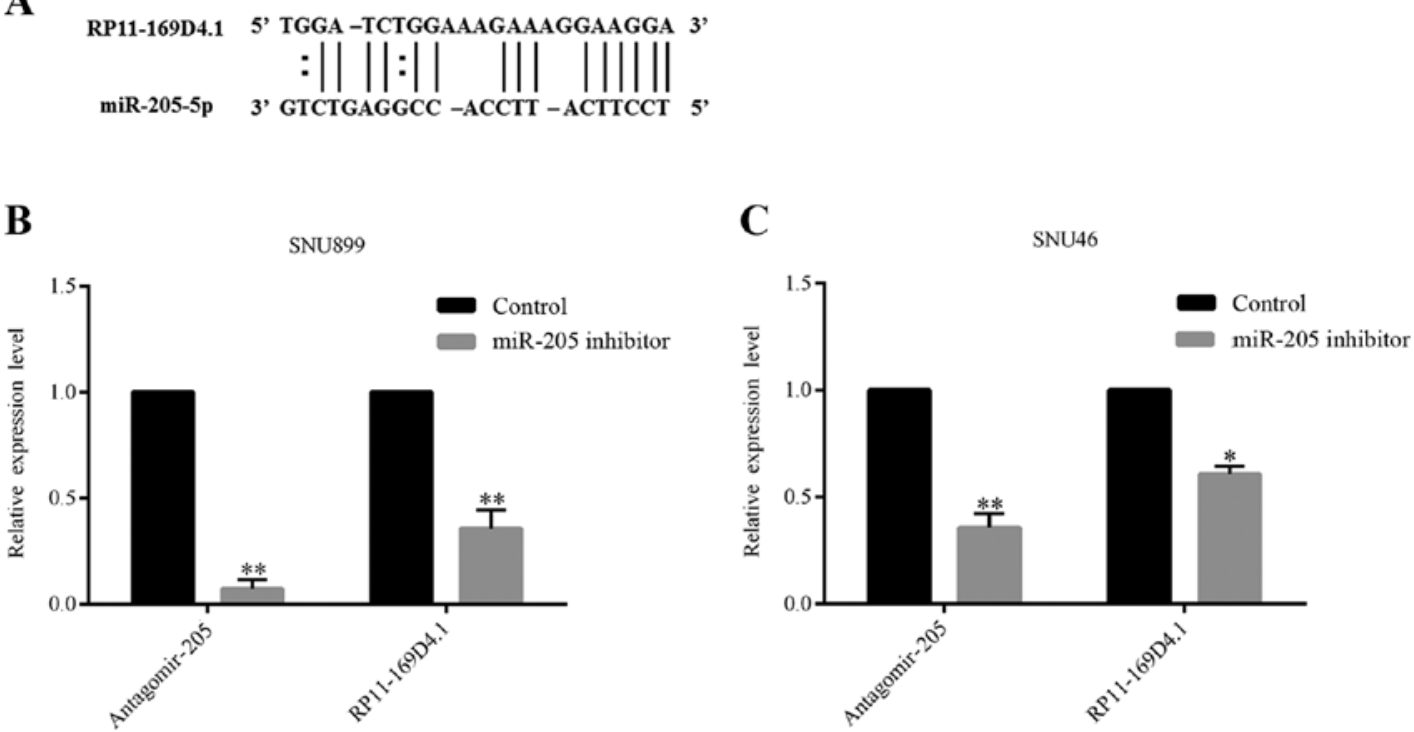

AGO2 complex immunoprecipitation

D

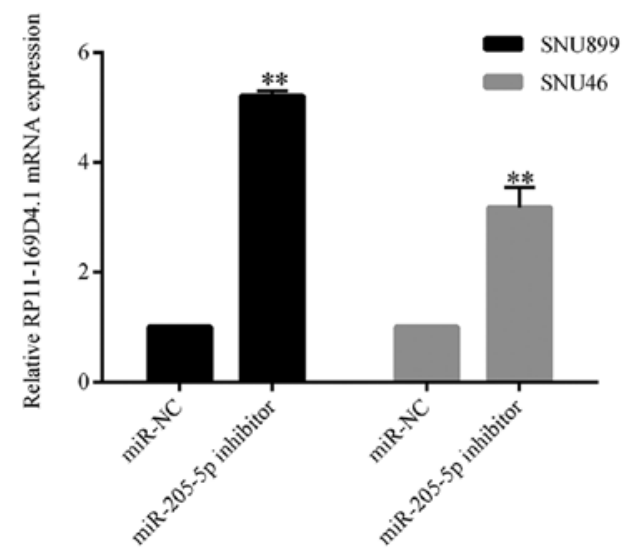

$\mathbf{E}$

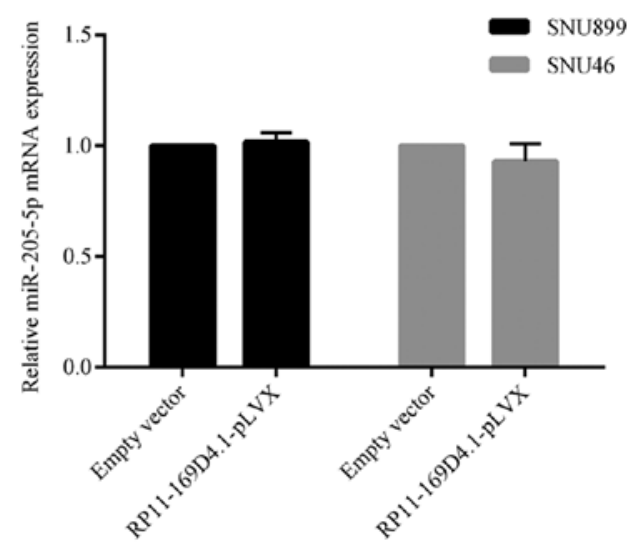

Figure 5. (A) Alignment of potential miR-205-5p base pairing with RP11-169D4.1 as identified by RNA22 program (https://cm.jefferson.edu/rna22/). (B and C) Relative expression level of miR-205-5p and RP11-169D4.1 mRNA in AGO2 complex from stable laryngeal carcinoma clone compromising miR-NC or miR-205-5p inhibitor. (D) With miR-205-5p suppression, RP11-169D4.1 mRNA level was remarkably increased. (E) With RP11-169D4.1 overexpression, the change of miR-205-5p mRNA level was not statistically significant. ${ }^{*} \mathrm{P}<0.05,{ }^{* *} \mathrm{P}<0.01$.

RP11-169D4.1 exerts its function via the AKT signaling pathway. According to studies that showed that loss of CDH1 could activate AKT signaling, we proposed that RP11-169D4.1 could regulate the AKT signaling pathway through CDH1. We detected the levels of AKT and phospho-AKT in LSCC cell lines. As expected, the level of p-AKT was reduced after cells were transfected with RP11-169D4.1-pLVX and total AKT levels were constant (Fig. 7C and D). These results suggest that RP11-169D4.1 may be a major player in the AKT signaling pathway.

\section{Discussion}

With the fast development of human genome and transcriptome sequencing technologies, much attention has been focused on lncRNA. IncRNAs could serve as potential diagnostic biomarkers and effective therapeutic targets. In the near future, in-depth research of IncRNAs is an attractive avenue to discover novel biomarkers or targets (22). To date, many studies have explored the various functions of lncRNAs in head and neck neoplasms (23). For example, the overexpression of UCA1 could promote the metastasis of TSCC cells (24). HOTAIR participated in PTEN methylation in Hep-2 cells (25) and had close correlation with miR-21 in LSCC (26). MALAT1 was a novel target of miR-217 and miR-101 and could stimulate the invasion and metastasis of ESCC cells (27).

It was observed that RP11-169D4.1 expression was downregulated in LSCC tissues and metastatic neck lymph nodes, and lower expression of RP11-169D4.1 predicted poor prognosis. In another study, the downregulation of RP11-169D4.1 in LSCC tissues was also observed (28). However, these studies were limited to its abnormal expression in LSCC. However, the specific function of RP11-169D4.1 and its potential mechanisms in LSCC still remain unknown.

In the present study, we first clarified the function of RP11-169D4.1, which was thought to play a tumor-suppressive role in LSCC. Moreover, RP11-169D4.1 expression was significantly correlated with LSCC metastasis to the neck 

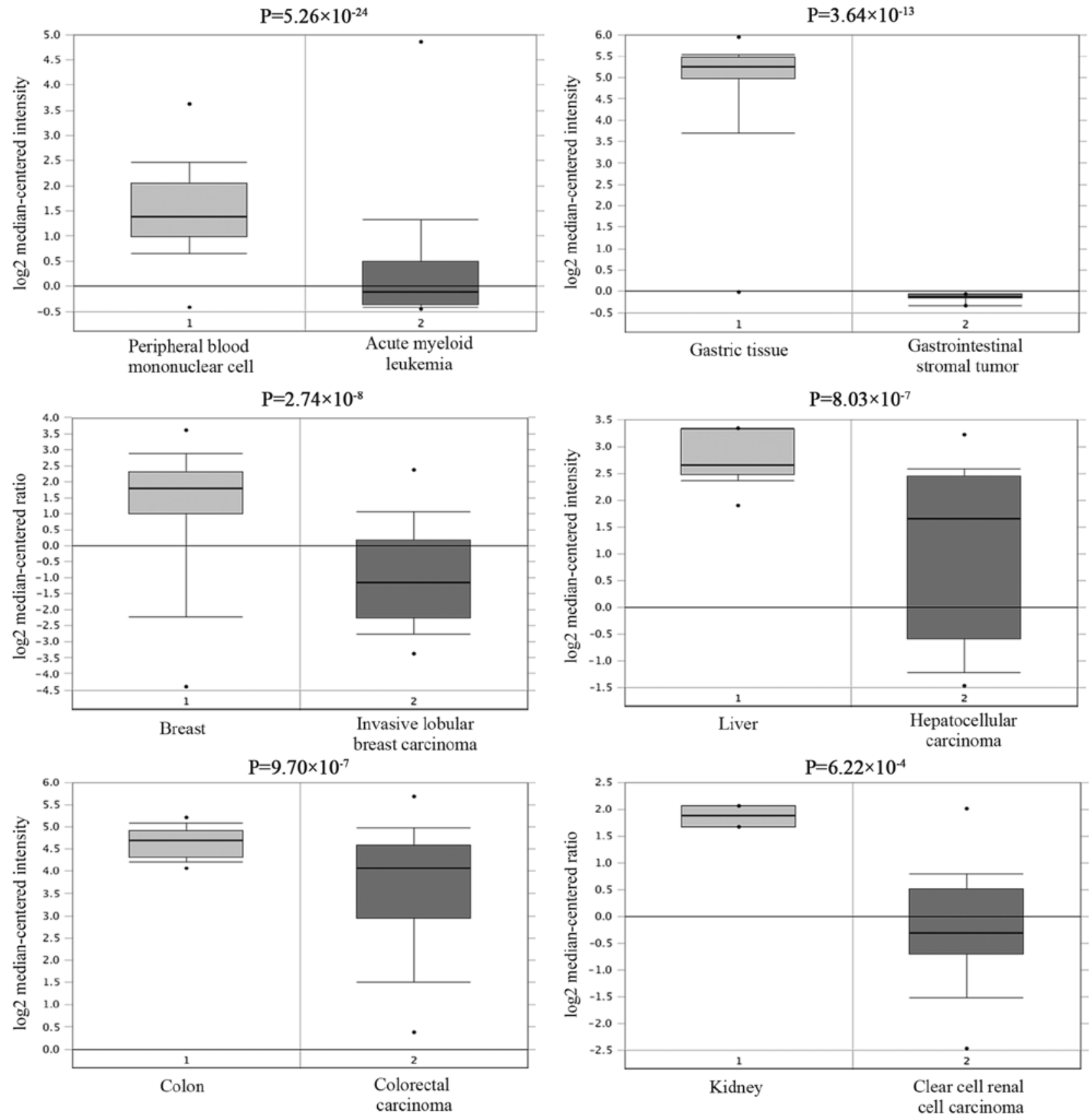

Figure 6. Box and whisker plots of Oncomine data on CDH1 mRNA levels (expressed as the $\log 2$ median-centered ratio) in various normal and cancerous tissues. P-values, Student's t-test.

lymph nodes. These data suggested that RP11-169D4.1 might participate in the metastasis of LSCC. The molecular mechanisms that control the expression of RP11-169D4.1 are now being elucidated. Through the bioinformatics tool RNA22, we know that RP11-169D4.1 might be a target of miR-205-5p. As an oncogenic microRNA, miR-205-5p has been well characterized for its role in LSCC (29). Some studies have indicated that miR-205-5p promotes the proliferation, migration and invasion of LSCC (30). Our findings highlight the interaction between miR-205-5p and the lncRNA RP11-169D4.1 during tumorigenesis and the progression of LSCC cells.

Next, we explored the molecular mechanisms underlying RP11-169D4.1 inhibition of EMT. EMT has been identi- fied as a paramount event in the early periods of metastatic dissemination in various tumor cells. During these periods, tumor cells become more active and have a stronger invasive ability (31). The CDH1 gene encodes E-cadherin, which is a transmembrane glycoprotein and a prototypical member of the classical cadherin family. E-cadherin/CDH1 plays a critical role in preserving cell polarity as well as maintaining epithelial integrity. It was reported that $\mathrm{CDH} 1$ expression was correlated with the metastasis to neck lymph nodes and the TNM stages in LSCC (32). As CDH1 is the most commonly expressed gene during EMT, we investigated whether RP11-169D4.1 could regulate the expression of $\mathrm{CDH}$.

To further explore the mechanisms of RP11-169D4.1, we tried to find the potential signaling pathway of RP11-169D4.1. 

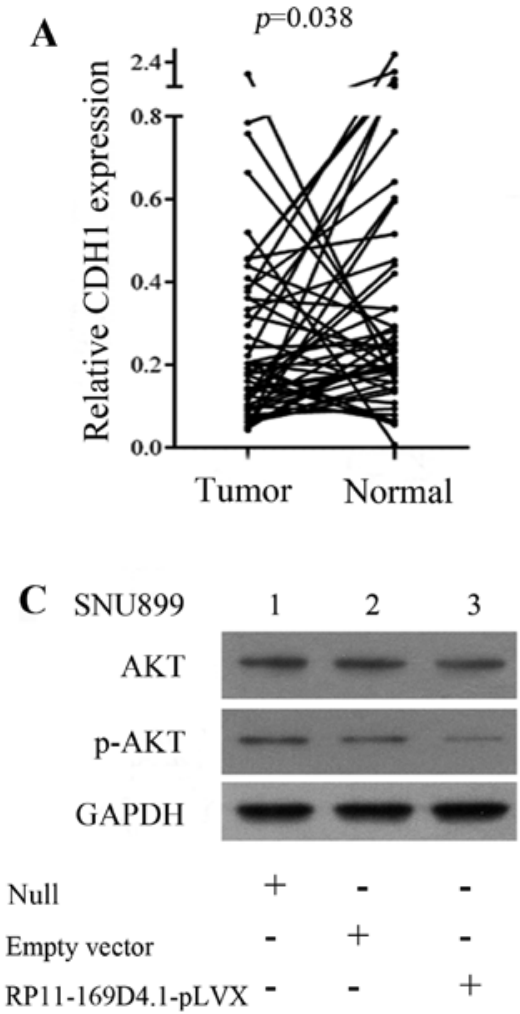
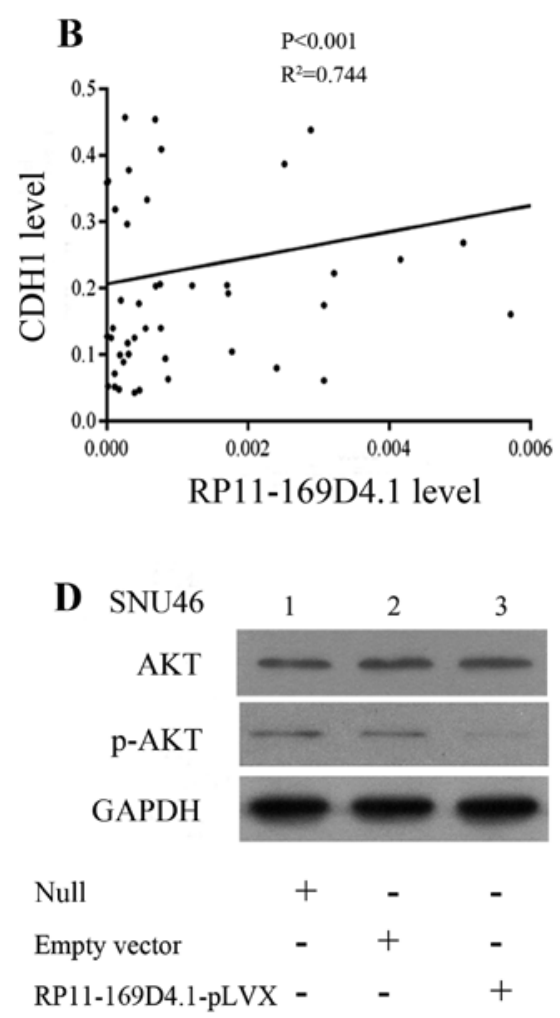

Figure 7. (A) Relative expression level of CDH1 mRNA in laryngeal tumor tissue samples and normal epithelial counterparts (n=51). (B) Correlation analysis of the expression of CDH1 and RP11-169D4.1 in LSCC cancer patients. (C and D) The protein levels of AKT and p-AKT in SNU899 and SNU46 transfected with empty vector or RP11-169D4.1-pLVX.

Previous studies have shown that CDH1 expression is regulated through the AKT pathway (33), and miR-205-5p promotes tumor metastasis by activating the AKT signaling (34), which indicates that RP11-169D4.1 might regulate the AKT signaling pathway by modulating CDH1. The AKT pathway is considered to be closely related to laryngeal carcinoma $(35,36)$. These results suggest that the miR-205-5p/RP11-169D4.1/CDH1/AKT signaling pathway may play an important role in the development of LSCC.

EMT is a process that results in the migration, invasion and metastasis of cancer cells. At the same time, loss of CDH1 (E-cadherin) is considered as a fundamental event in EMT. Several studies indicate that patients with lymph node metastasis tend to have higher recurrence rate and poor prognosis and RP11-169D4.1 can be considered as a predictor of lymph node metastasis in patients. However, the specific mechanism of regulating CDH1 for RP11-169D4.1 still needs further exploration. Continued study of these molecules and an improved understanding of the lncRNA RP11-169D4.1 will facilitate the development of more effective therapies against human laryngeal carcinoma.

In conclusion, the lncRNA RP11-169D4.1 is downregulated in LSCC and is associated with lymph node metastasis. Overexpression of RP11-169D4.1 in LSCC cells decreased cell migration and invasion in vitro. miR205-5p acted as the upstream molecule of RP11-169D4.1 activity. Furthermore, RP11-169D4.1 could suppress the process of EMT by modulating CDH1 expression. The miR-205-5p/RP11-169D4.1/CDH1/AKT signaling pathway is an important part of the molecular mechanisms of EMT in
LSCC. RP11-169D4.1 may be a novel and valuable therapeutic target in predicting outcomes of patients with LSCC.

\section{References}

1. Jemal A, Siegel R, Xu J and Ward E: Cancer statistics, 2010. CA Cancer J Clin 60: 277-300, 2010.

2. Yang Z, Meng Q, Luo J,Lu Q, Li X, Li G and Wan C: Development and validation of the simplified Chinese version of EORTC QLQ-H\&N35 for patients with head and neck cancer. Support Care Cancer 20: 1555-1564, 2012.

3. Hunter KD, Parkinson EK and Harrison PR: Profiling early head and neck cancer. Nat Rev Cancer 5: 127-135, 2005.

4. Marioni G, Marchese-Ragona R, Cartei G, Marchese F and Staffieri A: Current opinion in diagnosis and treatment of laryngeal carcinoma. Cancer Treat Rev 32: 504-515, 2006.

5. Denaro N, Merlano MC, Russi EG and Lo Nigro C: Non coding RNAs in head and neck squamous cell carcinoma (HNSCC): A clinical perspective. Anticancer Res 34: 6887-6896, 2014.

6. Rühle F and Stoll M: Long non-coding RNA databases in cardiovascular research. Genomics Proteomics Bioinformatics 14: 191-199, 2016.

7. Schmitt AM and Chang HY: Long noncoding RNAs in cancer pathways. Cancer Cell 29: 452-463, 2016.

8. Xu Y, Wang J, Qiu M, Xu L, Li M, Jiang F, Yin R and Xu L: Upregulation of the long noncoding RNA TUG1 promotes proliferation and migration of esophageal squamous cell carcinoma. Tumour Biol 36: 1643-1651, 2015.

9. Chen FJ, Sun M, Li SQ, Wu QQ, Ji L, Liu ZL, Zhou GZ, Cao G, Jin L, Xie HW, et al: Upregulation of the long non-coding RNA HOTAIR promotes esophageal squamous cell carcinoma metastasis and poor prognosis. Mol Carcinog 52: 908-915, 2013.

10. Chen D, Zhang Z, Mao C, Zhou Y, Yu L, Yin Y, Wu S, Mou X and Zhu Y: ANRIL inhibits $15^{\text {INK4b }}$ through the TGF $\beta 1$ signaling pathway in human esophageal squamous cell carcinoma. Cell Immunol 289: 91-96, 2014. 
11. Wang J, Qiu M, Xu Y, Li M, Dong G, Mao Q, Yin R and Xu L: Long noncoding RNA CCAT2 correlates with smoking in esophageal squamous cell carcinoma. Tumour Biol 36: 5523-5528, 2015.

12. Jia LF, Wei SB, Gan YH, Guo Y, Gong K, Mitchelson K, Cheng J and Yu GY: Expression, regulation and roles of miR-26a and MEG3 in tongue squamous cell carcinoma. Int J Cancer 135: 2282-2293, 2014.

13. Tong YS, Zhou XL, Wang XW, Wu QQ, Yang TX, Lv J, Yang JS, Zhu B and Cao XF: Association of decreased expression of long non-coding RNA LOC285194 with chemoradiotherapy resistance and poor prognosis in esophageal squamous cell carcinoma. J Transl Med 12: 233, 2014.

14. Gao T, He B, Pan Y, Xu Y, Li R, Deng Q, Sun H and Wang S: Long non-coding RNA 91H contributes to the occurrence and progression of esophageal squamous cell carcinoma by inhibiting IGF2 expression. Mol Carcinog 54: 359-367, 2015.

15. Shen Z, Li Q, Deng H, Lu D, Song H and Guo J: Long non-coding RNA profiling in laryngeal squamous cell carcinoma and its clinical significance: Potential biomarkers for LSCC. PLoS One 9: e108237, 2014.

16. Loher P and Rigoutsos I: Interactive exploration of RNA22 microRNA target predictions. Bioinformatics 28: 3322-3323, 2012.

17. Jiao F, Hu H, Han T, Zhuo M, Yuan C, Yang H, Wang L and Wang L: Aberrant expression of nuclear HDAC3 and cytoplasmic $\mathrm{CDH} 1$ predict a poor prognosis for patients with pancreatic cancer. Oncotarget 7: 16505-16516, 2016.

18. Wang Q, Wang B, Zhang YM and Wang W: The association between $\mathrm{CDH} 1$ promoter methylation and patients with ovarian cancer: A systematic meta-analysis. J Ovarian Res 9: 23, 2016.

19. Berx G, Cleton-Jansen AM, Nollet F, de Leeuw WJ, van de Vijver M, Cornelisse C and van Roy F: E-cadherin is a tumour/invasion suppressor gene mutated in human lobular breast cancers. EMBO J 14: 6107-6115, 1995.

20. Zhou J, Tao D, Xu Q, Gao Z and Tang D: Expression of E-cadherin and vimentin in oral squamous cell carcinoma. Int J Clin Exp Pathol 8: 3150-3154, 2015.

21. Kowalski PJ, Rubin MA and Kleer CG: E-cadherin expression in primary carcinomas of the breast and its distant metastases. Breast Cancer Res 5: R217-R222, 2003.

22. Schmitz SU, Grote P and Herrmann BG: Mechanisms of long noncoding RNA function in development and disease. Cellular and molecular life sciences. Cell Mol Life Sci 73: 2491-2509, 2016.

23. Chen H, Xin Y, Zhou L, Huang JM, Tao L, Cheng L and Tian J: Cisplatin and paclitaxel target significant long noncoding RNAs in laryngeal squamous cell carcinoma. Med Oncol 31: 246, 2014.
24. Fang Z, Wu L, Wang L, Yang Y, Meng Y and Yang H: Increased expression of the long non-coding RNA UCA1 in tongue squamous cell carcinomas: A possible correlation with cancer metastasis. Oral Surg Oral Med Oral Pathol Oral Radiol 117: 89-95, 2014.

25. Li D, Feng J, Wu T, Wang Y, Sun Y, Ren J and Liu M: Long intergenic noncoding RNA HOTAIR is overexpressed and regulates PTEN methylation in laryngeal squamous cell carcinoma. Am J Pathol 182: 64-70, 2013.

26. Wang J, Zhou Y, Lu J, Sun Y, Xiao H, Liu M and Tian L: Combined detection of serum exosomal miR-21 and HOTAIR as diagnostic and prognostic biomarkers for laryngeal squamous cell carcinoma. Med Oncol 31: 148, 2014.

27. Wang X, Li M, Wang Z, Han S, Tang X, Ge Y, Zhou L, Zhou C, Yuan $Q$ and Yang M: Silencing of long noncoding RNA MALAT1 by miR-101 and miR-217 inhibits proliferation, migration, and invasion of esophageal squamous cell carcinoma cells. J Biol Chem 290: 3925-3935, 2015.

28. Zou AE, Ku J, Honda TK, Yu V, Kuo SZ, Zheng H, Xuan Y, Saad MA, Hinton A, Brumund KT, et al: Transcriptome sequencing uncovers novel long noncoding and small nucleolar RNAs dysregulated in head and neck squamous cell carcinoma. RNA 21: 1122-1134, 2015.

29. Cao P, Zhou L, Zhang J, Zheng F, Wang H, Ma D and Tian J: Comprehensive expression profiling of microRNAs in laryngeal squamous cell carcinoma. Head Neck 35: 720-728, 2013.

30. Tian L, Zhang J, Ge J, Xiao H, Lu J, Fu S, Liu M and Sun Y: MicroRNA-205 suppresses proliferation and promotes apoptosis in laryngeal squamous cell carcinoma. Med Oncol 31: 785, 2014.

31. Thiery JP, Acloque H, Huang RY and Nieto MA: Epithelialmesenchymal transitions in development and disease. Cell 139: 871-890, 2009.

32. Ahmed RA, Shawky AA and Hamed RH: Prognostic significance of cyclin D1 and E-cadherin expression in laryngeal squamous cell carcinoma. Pathol Oncol Res 20: 625-633, 2014.

33. Liu X, Su L and Liu X: Loss of CDH1 up-regulates epidermal growth factor receptor via phosphorylation of YBX1 in non-small cell lung cancer cells. FEBS Lett 587: 3995-4000, 2013.

34. Mao Y, Wu S, Zhao R and Deng Q: MiR-205 promotes proliferation, migration and invasion of nasopharyngeal carcinoma cells by activation of AKT signalling. J Int Med Res 44: 231-240, 2016.

35. Lu B, Di W, Wang H, Ma H, Li J and Zhang Q: Tumor suppressor TSLC1 is implicated in cell proliferation, invasion and apoptosis in laryngeal squamous cell carcinoma by regulating Akt signaling pathway. Tumour Biol 33: 2007-2017, 2012.

36. Yang N, Hui L, Wang Y, Yang H and Jiang X: SOX2 promotes the migration and invasion of laryngeal cancer cells by induction of MMP-2 via the PI3K/Akt/mTOR pathway. Oncol Rep 31: 2651-2659, 2014. 